\title{
Commentary
}

\section{Does WHI tell us how to prevent diabetes?}

Diabetes is occurring in epidemic proportions around the globe. Using the WHO global database, King and colleagues have estimated that the number of adults with diabetes will increase from 135 million in 1995 to 300 million in 2025 , with a predicted world diabetes prevalence of 5.4\% [1]. Most of these future cases are expected to occur in the developing world, but the prevalence of self-reported diabetes in the United States has already increased, from $4.9 \%$ in 1990 to $7.3 \%$ in 2000 [2]. Women are more likely to develop diabetes [2], and since women with diabetes have not experienced the same reduction in cardiovascular outcomes as men with diabetes in recent years [3], it can be a particularly devastating disease for them.

Treating diabetes has improved health outcomes and quality of life, but prevention of the disease will have a much greater impact on public health. The Diabetes Prevention Program (DPP) found that intensive lifestyle interventions, with the aim of modest weight reduction and 150 minutes of exercise a week, considerably reduced the number of high-risk subjects who developed diabetes over a three-year period [4], but translation of these findings into the clinical setting has been difficult. Health care systems have been slow to invest in the labour-intensive systems necessary to support patients as they change their lifestyles. Also, very few patients have succeeded in maintaining weight loss over the longer term without the type of support available in the DPP. Medications such as metformin, troglitazone and acarbose have also been studied in terms of diabetes prevention $[4,5,6]$. Although effective, they have had significantly less impact than intensive lifestyle changes alone and may simply postpone the inevitable presentation of diabe-

Received: 11 May 2004 / Accepted: 16 June 2004

Published online: 7 July 2004

(C) Springer-Verlag 2004 tes in individuals at risk. Additional strategies to prevent diabetes are clearly needed.

This issue of the journal features a report from the Women's Health Initiative (WHI) that offers new insights into how diabetes may be prevented in women [7]. The study was designed to examine the effect of post-menopausal hormone replacement on a number of health outcomes, with a primary endpoint of coronary heart disease death or non-fatal myocardial infarction. More than 15,000 women were randomised to receive either $0.625 \mathrm{mg}$ of conjugated equine oestrogens and $2.5 \mathrm{mg}$ of medroxyprogesterone acetate or placebo each day. The study was halted after an average of 5.6 years of follow-up because of adverse events in the intervention arm. Evaluation of the data after the end of the study did, however, reveal that women randomised to hormone therapy had a lower incidence of self-reported diabetes than those randomised to placebo. The cumulative incidence of treated diabetes was $3.5 \%$ in the hormone treated group vs $4.2 \%$ in the control group, with a hazard ratio of 0.79 (nominal 95\% CI 0.67-0.93). Interestingly, the hazard ratio was unaffected by adjustment for changes in body mass index or waist circumference.

Whilst confirming the secondary endpoint observations made by the Heart and Estrogen/Progestin Replacement Study (HERS) in post-menopausal women with coronary disease [8], Margolis and colleagues were able to progress towards an understanding of the mechanisms involved, in that a subset of their participants also had blood obtained for fasting glucose and insulin at baseline and during follow-up. This randomly selected cohort represented $8.6 \%$ of the population and was stratified by age, clinical centre and ethnicity to oversample minority women. At one year of follow-up, HOMA-IR, a measure of insulin sensitivity calculated from fasting levels of glucose and insulin [9], was significantly lower in women on hormone replacement. 
By Year 3, this difference in HOMA-IR had disappeared, probably because of lower numbers and poorer adherence to the hormone regimen, but the observations provide evidence that reduced insulin resistance may be the mechanism by which hormone replacement therapy reduced the incidence of diabetes in this group.

Why should hormone replacement therapy reduce insulin resistance? It is unlikely that progestin alone is responsible, since such agents have been shown to be associated with an increase in the incidence of diabetes in women using the drug for contraception [10]. Oestrogen alone might be responsible, but conclusions cannot be reached until all of the data from the WHI Estrogen alone study can be analysed [11]. Oestrogen is, however, known to increase serum sex hormone binding globulin (SHBG), thereby reducing free testosterone concentrations and increasing insulin sensitivity in post-menopausal women [12].

Does this imply that women at risk of getting diabetes should be treated with oestrogen? Probably not, given the increased risk of stroke and pulmonary embolism seen in women treated with oestrogen alone in the WHI [11]. But these observations do suggest that a better understanding of how oestrogen alters insulin sensitivity could lead to new therapies for use in the prevention of diabetes. Although the selective oestrogen receptor modulator, raloxifene, does not appear to modify insulin sensitivity in the same way as oestrogen [13], further investigation of drugs with oestrogenic qualities could lead to an agent that improves insulin sensitivity without increasing the risk of cancer or cardiovascular events.

Meanwhile, the WHI has shown that post-menopausal women randomised to hormone replacement therapy are less likely to progress to diabetes, possibly because of reduced insulin resistance. The effect on insulin resistance appeared unrelated to changes in body mass index, but randomised trials like the Diabetes Prevention Project [4] or Finnish Diabetes Prevention Study [14] suggest that lowering insulin resistance through weight reduction and/or increased exercise also reduces the incidence of diabetes. So while the search for useful therapies continues, women and men at risk of diabetes would be well advised to continue to eat less and exercise more.

\author{
E. R. Seaquist ( $)$ \\ Division of Endocrinology and Diabetes, \\ Department of Medicine, \\ University of Minnesota Medical School, MMC 101, \\ 420 Delaware St SE, Minneapolis, MN 55455, USA \\ E-mail: seaqu001@umn.edu
}

\section{References}

1. King H, Aubert RE, Herman WH (1998) Global burden of diabetes, 1995-2025: Prevalence, numerical estimates, and projections. Diabetes Care 21:1414-1431

2. Mokdad AH, Bowman BA, Ford ES, Vinicor F, Marks JS, Koplan JP (2001) The continuing epidemics of obesity and diabetes in the United States. JAMA 286:1195-1200

3. Gu K, Cowie CC, Harris MI (1999) Diabetes and decline in heart disease mortality in US adults. JAMA 281:12911297

4. Knowler WC, Barrett-Connor E, Fowler SE et al. (2002) Reduction in the incidence of type 2 diabetes with lifestyle intervention or metformin. N Engl J Med 346:393403

5. Chiasson JL, Josse RG, Gomis R, Hanefeld M, Karasik A, Laakso M (2002) Acarbose for prevention of type 2 diabetes mellitus: the STOP-NIDDM randomised trial. Lancet 359:2072-2077

6. Buchanan TA, Xiang AH, Peters RK et al. (2002) Preservation of pancreatic beta-cell function and prevention of type 2 diabetes by pharmacological treatment of insulin resistance in high-risk Hispanic women. Diabetes 51:27962803

7. Margolis KL, Bonds DE, Rodabough RJ et al. (2004) Effect of oestrogen plus progestin on the incidence of diabetes in postmenopausal women: Results from the Women's Health Initiative Hormone Trial. Diabetologia 47: dx.doi.org/10.1007/s00125-004-1448-x

8. Kanaya A, Herrington D, Vittinghoff E et al. (2003) Glycemic effects of postmenopausal hormone therapy: the heart and estrogen/progestin replacement study. Ann Intern Med 138:1-9

9. Matthews DR, Hosker JP, Rudenski AS, Naylor BA, Treacher DF, Turner RC (1985) Homeostasis model assessment: Insulin resistance and beta-cell function from fasting plasma glucose and insulin concentrations in man. Diabetologia 28:412-419

10. Kim C, Seidel KW, Begier EA, Kwok YS (2001) Diabetes and depot medroxyprogesterone contraception in Navajo women. Arch Intern Med 161:1766-1771

11. Anderson GL, Limacher M, Assaf AR et al. (2004) Effects of conjugated equine estrogen in postmenopausal women with hysterectomy: the Women's Health Initiative randomized controlled trial. JAMA 291:1701-1712

12. Andersson B, Mattsson LA, Hahn L et al. (1997) Estrogen replacement therapy decreases hyperandrogenicity and improves glucose homeostasis and plasma lipids in postmenopausal women with noninsulin-dependent diabetes mellitus. J Clin Endocrinol Metab 82:638-643

13. Andersson B, Johannsson G, Holm G et al. (2002) Raloxifene does not affect insulin sensitivity or glycemic control in postmenopausal women with type 2 diabetes mellitus: a randomized clinical trial. J Clin Endocrinol Metab 87:122-128

14. Tuomilehto J, Lindstrom J, Eriksson JG et al. (2001) Prevention of type 2 diabetes mellitus by changes in lifestyle among subjects with impaired glucose tolerance. N Engl J Med 344:1343-1350 